\title{
Glutamatergic neurometabolite levels in major depressive disorder: a systematic review and meta-analysis of proton magnetic resonance spectroscopy studies
}

\author{
Sho Moriguchi ${ }^{1,2} \cdot$ Akihiro Takamiya $^{1} \cdot$ Yoshihiro Noda $^{1} \cdot$ Nobuyuki Horita $^{3} \cdot$ Masataka Wada $^{1}$ Sakiko Tsugawa ${ }^{1}$ \\ Eric Plitman ${ }^{2} \cdot$ Yasunori Sano $^{1} \cdot$ Ryosuke Tarumi $^{1} \cdot$ Muhammad ElSalhy $^{1} \cdot$ Nariko Katayama $^{1} \cdot$ Kamiyu Ogyu $^{1}$. \\ Takahiro Miyazaki $^{1}$ - Taishiro Kishimoto ${ }^{1}$ - Ariel Graff-Guerrero ${ }^{2}$ Jeffrey H. Meyer ${ }^{2}$ - Daniel M. Blumberger ${ }^{4}$. \\ Zafiris J. Daskalakis ${ }^{4}$ Masaru Mimura ${ }^{1} \cdot$ Shinichiro Nakajima ${ }^{1,2}$
}

Received: 5 May 2018 / Revised: 13 July 2018 / Accepted: 10 August 2018 / Published online: 12 October 2018

(c) The Author(s) 2018. This article is published with open access

\begin{abstract}
Alterations in glutamatergic neurotransmission are implicated in the pathophysiology of depression, and the glutamatergic system represents a treatment target for depression. To summarize the nature of glutamatergic alterations in patients with depression, we conducted a meta-analysis of proton magnetic resonance $\left({ }^{1} \mathrm{H}-\mathrm{MRS}\right)$ spectroscopy studies examining levels of glutamate. We used the search terms: depress* AND (MRS OR "magnetic resonance spectroscopy"). The search was performed with MEDLINE, Embase, and PsycINFO. The inclusion criteria were ${ }^{1} \mathrm{H}-\mathrm{MRS}$ studies comparing levels of glutamate + glutamine (Glx), glutamate, or glutamine between patients with depression and healthy controls. Standardized mean differences (SMD) were calculated to assess group differences in the levels of glutamatergic neurometabolites. Fortynine studies met the eligibility criteria, which included 1180 patients and 1066 healthy controls. There were significant decreases in Glx within the medial frontal cortex (SMD $=-0.38 ; 95 \% \mathrm{CI},-0.69$ to -0.07 ) in patients with depression compared with controls. Subanalyses revealed that there was a significant decrease in Glx in the medial frontal cortex in medicated patients with depression $(\mathrm{SMD}=-0.50 ; 95 \% \mathrm{CI},-0.80$ to -0.20 ), but not in unmedicated patients $(\mathrm{SMD}=-0.27 ; 95 \% \mathrm{CI},-0.76$ to 0.21$)$ compared with controls. Overall, decreased levels of glutamatergic metabolites in the medial frontal cortex are linked with the pathophysiology of depression. These findings are in line with the hypothesis that depression may be associated with abnormal glutamatergic neurotransmission.
\end{abstract}

These authors contributed equally: Sho Moriguchi, Akihiro Takamiya

Electronic supplementary material The online version of this article (https://doi.org/10.1038/s41380-018-0252-9) contains supplementary material, which is available to authorized users.

Yoshihiro Noda

yoshi-tms@keio.jp

1 Department of Neuropsychiatry, Keio University School of Medicine, Tokyo, Japan

2 Research Imaging Centre, Centre for Addiction and Mental Health, University of Toronto, Toronto, Canada

3 Department of Pulmonology, Yokohama City University Graduate School of Medicine, Yokohama, Japan

4 Temerty Centre for Therapeutic Brain Intervention, Centre for Addiction and Mental Health, Department of Psychiatry, University of Toronto, Toronto, Canada

\section{Introduction}

The heterogeneity of the illness features that characterize depression makes it difficult to elucidate the underlying pathology of the illness and its treatment. The glutamate hypothesis of depression was proposed in the 1990s, when antagonists of the $N$-methyl-D-aspartate (NMDA) receptor, an ionotropic glutamate receptor, were found to possess antidepressant-like mechanisms of action in mice [1]. Furthermore, infusion of low-dose ketamine, which is an NMDA receptor antagonist, is associated with robust decreases in depressive symptoms in depressed patients [2].

Recent data suggest that glutamatergic dysfunction is involved in the biological mechanisms underlying depression [3]. For example, positron emission tomography (PET) studies have reported reduced metabotropic glutamate receptor subtype 5 density in patients with depression, which was further substantiated by a post-mortem study [4]. 
In addition, animal model studies have also demonstrated that depressive-like behaviors are associated with alterations in cortical glutamate [5-7]. Furthermore, a recent metaanalysis showed that ketamine has rapid antidepressant effects in depressed patients compared with placebo [8]. As a result, glutamatergic neurometabolites have garnered increasing interest in terms of their role in the underlying pathophysiology of depression.

To date, several proton magnetic resonance spectroscopy $\left({ }^{1} \mathrm{H}-\mathrm{MRS}\right)$ studies have examined regional levels of glutamatergic metabolites in patients with depression compared with controls. However, findings are inconsistent across studies, which report increases [8], no differences [9, 10], or decreases [11-13] in glutamatergic neurometabolite levels in patients with depression across a variety of brain regions. These differences may be due to differences in regions of interest (ROIs), MRS methodologies, stages or severities of illness, or medications (e.g., antidepressant treatments). A recent meta-analysis noted that glutamate levels were lower within the anterior cingulate cortex (ACC) of patients with depression compared with controls [14]; of note, this metaanalysis included 16 studies published until 2010 and consisted of 281 patients and 301 controls. There were some limitations in this meta-analysis, as several of the included studies had all of the ROIs merged into one [14]. Furthermore, another recent meta-analysis reported that glutamine + glutamate (Glx) levels were decreased in the prefrontal cortex (PFC) in patients with depression compared with controls, whereas no significant difference was found in terms of glutamate levels between the two groups [15]. This meta-analysis also noted that reductions in Glx levels within the PFC were related to the number of failed antidepressant treatment trials. Specifically, this metaanalysis focused exclusively on glutamate and Glx in the PFC, and included 17 studies published until 2014, totaling 363 patients and 306 controls [15]. Notably, the inclusion of more recent reports would allow the meta-analysis of data from specific brain regions in patients with depression. Importantly, 22 papers, which is more than double the number of studies included in past meta-analyses, have been published since these meta-analyses.

Therefore, we conducted a systematic review and metaanalysis to compare the levels of specific regional glutamatergic neurometabolites; we aimed to do so in a comprehensive fashion, including the most recent studies on the topic. Based on previous meta-analyses, we hypothesized that Glx levels in the medial PFC (mPFC) would be decreased in patients with depression compared with controls [14, 15]. We also explored the influences of age, sex, symptom severity, and antidepressant treatment on group differences in the levels of regional glutamatergic neurometabolites.

\section{Methods}

\section{Protocol registration}

The full protocol was uploaded to the International Prospective Register of Systematic Reviews website (CRD42017079668). We have followed the Preferred Reporting Items for Systematic Reviews and MetaAnalyses (PRISMA) statement [16].

\section{Study search}

We used the search terms: depress* AND (MRS OR "magnetic resonance spectroscopy"). The search was performed with MEDLINE (1946 to October 2017), Embase (1947 to October 2017), and PsycINFO (1806 to October 2017). The searches were rerun just before the final analyses and further studies were retrieved for inclusion in March 2018.

A hand search was conducted by SM, AT, and SN. Candidate articles were independently screened and scrutinized by these authors. Discrepancies in study selection were resolved by discussion among the three authors.

\section{Data extraction}

Any data concerning a fundamental description of each study and data related to the outcomes described below were independently extracted by SM and AT. We have extracted any data regardless of the definitions of the primary/secondary endpoints of each original study. The extracted data were cross-checked and discrepancies were resolved by discussion between the two authors. If different publications reported data from the same population, we included data from the publication with the larger sample size. When studies did not report data, we e-mailed the authors to obtain the data.

\section{Inclusion criteria}

\section{Publication type}

Any English, full-length, or short articles were included, whereas non-English articles and conference abstracts were excluded.

\section{Study design}

We included cross-sectional studies and randomized control studies with MRS data in both patients with depression and healthy controls. 


\section{Patients}

Studies were included if: (1) patients met the Diagnostic and Statistical Manual of Mental Disorders (DSM), 3rd, 4th, or 5th edition criteria for major depressive disorder without bipolar disorder, the International Classification of Disease diagnostic (ICD) criteria for major depressive disorder without bipolar disorder, or consensus expert evaluation confirmed the diagnosis of depression without bipolar disorder; (2) we included all age range from pediatric to senior subjects; (3) authors compared glutamate, glutamine, or glutamine + glutamate (Glx) levels in the brains of patients with depression and subjects without depression using ${ }^{1} \mathrm{H}$ MRS; (4) authors included at least three subjects in each group; and (5) data were sufficient and appropriate to obtain mean differences between groups. In contrast, studies were excluded if they did not present data exclusively from patients with depression.

\section{Quality assessment}

The quality of the original studies was assessed using the Newcastle-Ottawa Quality Assessment Scale after arranging it for a cross-sectional study design [17]. This scale assigns four and two points for patient selection and comparability, respectively. Six points indicated the highest quality, whereas zero points indicates the lowest quality.

\section{Primary outcomes}

The primary outcome was Glx levels. The secondary outcomes were glutamate and glutamine levels. The ROIs were as follows: (1) the mPFC, including both the mPFC and ACC since their ROIs often overlap; (2) the dorsolateral PFC (DLPFC); (3) the thalamus; (4) the medial temporal lobe (mTemp), including the hippocampus and para hippocampus; and (5) the occipital cortex. When data from bilateral lobes were reported separately, the left lobe was used because the left lobe was examined in most studies.

\section{Statistical analyses}

All continuous primary and secondary outcomes were compared between patients and controls using the standardized mean difference (SMD). SMD and two-sided $95 \%$ confidence intervals (CIs) were chosen as the summary statistic for the meta-analysis. Interpretation of the magnitude of the SMD was as follows: small, SMD $=0.2$; medium, $\mathrm{SMD}=0.5$; and large, $\mathrm{SMD}=0.8$. The calculation of SMD was conducted using Review Manager ver. 5.3 (Cochrane Collaboration, Oxford, UK). We conducted a meta-analysis for each metabolite in each ROI that included four studies or more. The heterogeneity among original studies and subgroups was evaluated using the $I^{2}$ statistic, whereby $I^{2}=0 \%$ indicated no heterogeneity, $0 \%<I^{2}<30 \%$ indicated the least heterogeneity, $30 \% \leq I^{2}<50 \%$ indicated moderate heterogeneity, $50 \% \leq I^{2}<75 \%$ indicated substantial heterogeneity, and $75 \% \leq I^{2}$ indicated considerable heterogeneity. Publication bias was evaluated using a funnel plot and Begg-Kendall test. Subgroup analyses based on medication status (i.e., unmedicated, medicated) were performed for levels of glutamatergic neurometabolites. To make these subgroups, a clear cut-off year for the unmedicated period was not set. If there were four or less studies on one ROI, the ROI was not included in the analysis. We used a meta-regression in mixed-effects model to assess the relationship between moderators and the effect size of glutamatergic neurometabolites between patients and controls as a dependent variable. For the metaregression, we used "average age among subjects", "female ratios among subjects", or "depression severity with 17 items Hamilton Depression Rating Scale (HAMD-17)" as independent variables, because the HAMD-17 was examined in most studies as the severity scale. A mixed-model meta-regression was performed using the Comprehensive Meta-Analysis version 3. The variables for each study included: (1) clinico-demographic characteristics of the subjects (i.e., age, sex, medication status, and symptom severity, as measured by the HAMD, Beck's Depression Inventory (BDI), or the Montgomery-Asberg Depression Rating Scale (MADRS)); (2) MRS scan methods; (3) neurometabolite quantification methods; and (4) ROIs. Given our initial assumption that Glx levels in the mPFC would be decreased in patients with depression compared with controls, the significance level for all tests was set at a $p$-value of 0.05 (two tailed).

\section{Results}

\section{Characteristics of included studies}

The search identified 49 studies, which included a total of 1180 patients and 1066 healthy controls. A total of 49 articles were ultimately included into our analysis (Fig. 1). Characteristics of the studies are described in Table 1. Thirty-six studies (73\%) examined Glx, 27 studies $(55 \%)$ examined glutamate, and 11 studies (22\%) examined glutamine. Twenty-nine studies (59\%) and 19 studies $(39 \%)$ reported on unmedicated and medicated patients, respectively. Three studies measured glutamine levels in the DLPFC. Two studies measured Glx levels in the occipital cortex and thalamus, glutamate levels in the occipital cortex, and glutamine levels in the mTemp. One study measured glutamate levels in the thalamus and 


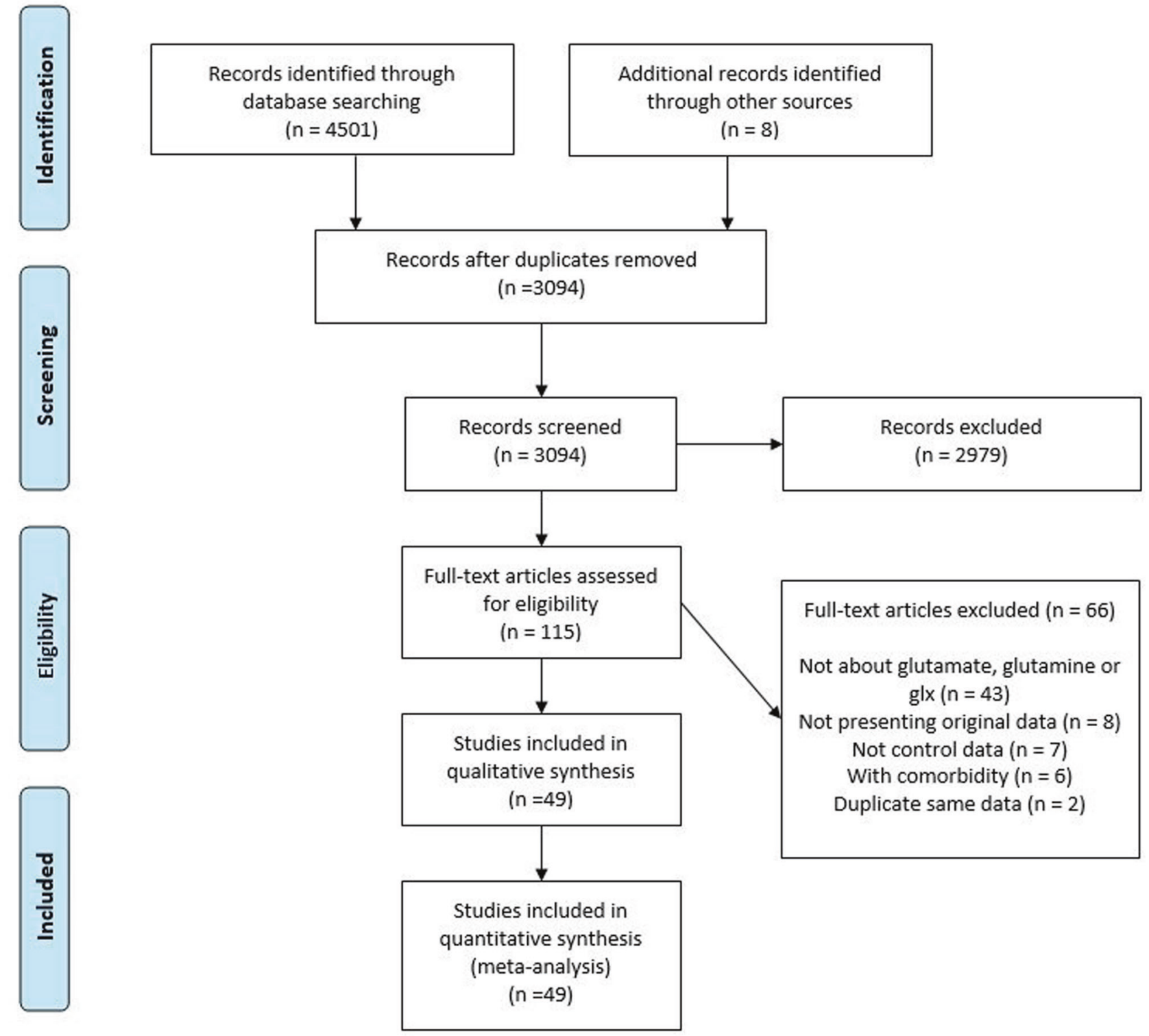

Fig. 1 Preferred reporting items for systematic reviews and meta-analyses (PRISMA) diagram for study search

glutamine levels in the occipital cortex and thalamus. The sample sizes ranged from 9 to 63 for patients with depression and 10 to 50 for healthy controls. The average ages of each study ranged from 13.3 to 72.1 years for patients with depression and 13.6 to 72.7 years for healthy controls. Thirty-two $(65 \%)$ and $12(24 \%)$ studies were performed at a magnetic field strength of $3 \mathrm{~T}$ and $1.5 \mathrm{~T}$, respectively. Magnetic resonance imaging (MRI) protocols and methodological information, including measurement technique and parameters, for each study are described in Table 1. The Newcastle-Ottawa Scale score ranged from 2 to 6 and the average was 5.1
(Supplementary Table 1), which suggests that the quality of the included studies was good on average.

\section{Meta-analysis}

Glx levels in the mPFC were measured in 502 patients and 408 controls. There were significantly lower levels of Glx within the medial frontal cortex in patients with depression compared with controls (SMD $=-0.38 ; 95 \% \mathrm{CI},-0.69$ to $-0.07 ; I^{2}=81 \% ; p=0.2$ ) (Table 2, Fig. 2). There were no identified differences in glutamate or glutamine levels in any regions (Table 2, Fig. 3 and Supplementary Figure 2). 


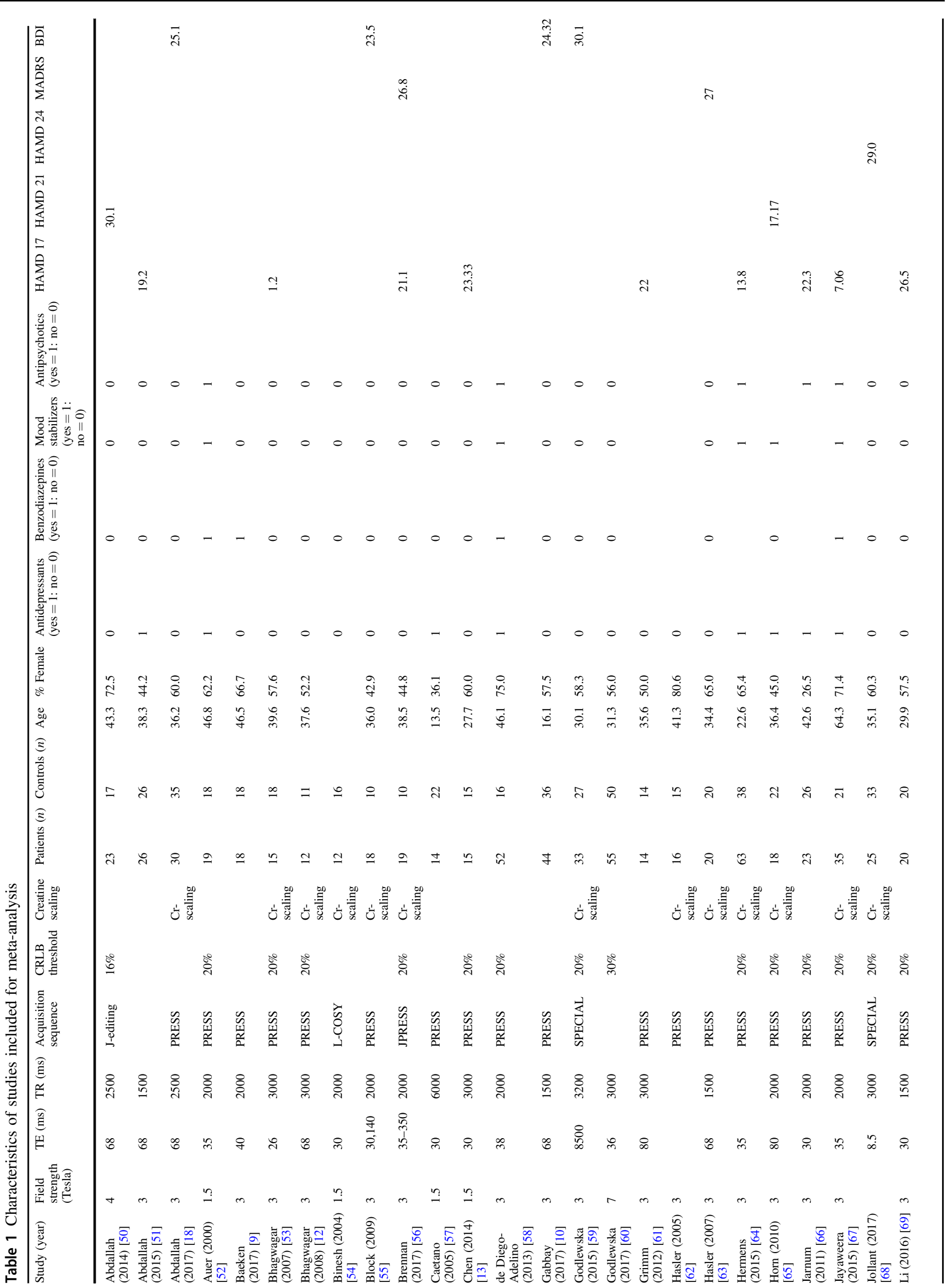




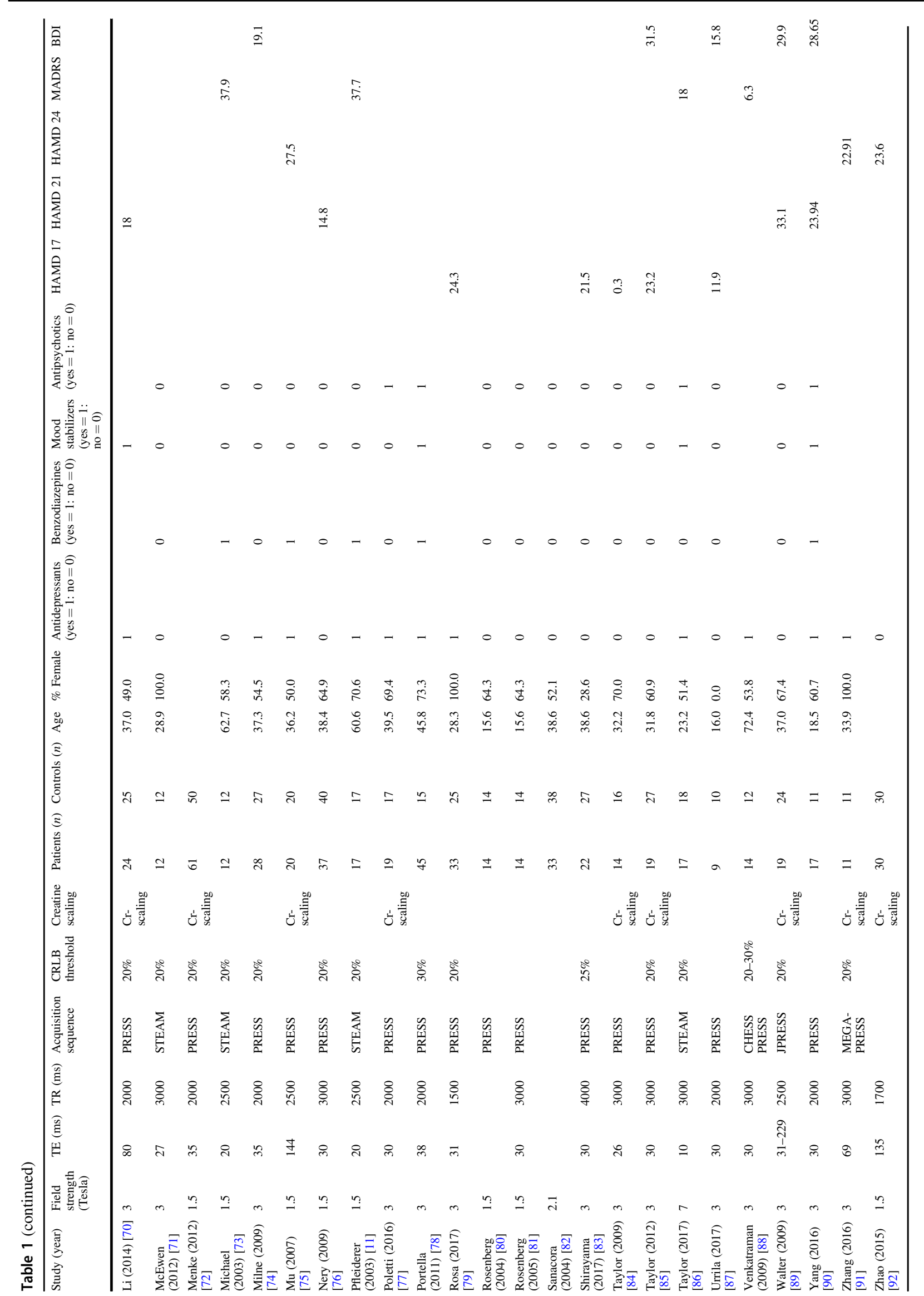


Table 2 Meta-analysis results summary for depression and controls in all brain regions

\begin{tabular}{|c|c|c|c|c|c|c|}
\hline Random effect model & Study number & Depression $(n)$ & Controls $(n)$ & SMD $(95 \%$ CI $)$ & $p$-Value & $I^{2}$ \\
\hline \multicolumn{7}{|l|}{ Glx } \\
\hline Medial prefrontal & 25 & 502 & 488 & $-0.38(-0.69$ to -0.07$)$ & 0.02 & $81 \%$ \\
\hline Dorsolateral prefrontal & 10 & 184 & 180 & $-0.36(-0.76$ to 0.04$)$ & 0.08 & $70 \%$ \\
\hline Medial temporal & 8 & 244 & 189 & $-0.10(-0.48$ to 0.29$)$ & 0.63 & $72 \%$ \\
\hline \multicolumn{7}{|l|}{ Glutamate } \\
\hline Medial prefrontal & 18 & 371 & 359 & $-0.19(-0.60$ to 0.22$)$ & 0.35 & $85 \%$ \\
\hline Dorsolateral prefrontal & 7 & 156 & 161 & $0.13(-0.28$ to 0.55$)$ & 0.53 & $68 \%$ \\
\hline Medial temporal & 5 & 195 & 148 & $0.01(-0.37$ to 0.39$)$ & 0.95 & $64 \%$ \\
\hline \multicolumn{7}{|l|}{ Glutamine } \\
\hline Medial prefrontal & 8 & 170 & 174 & $0.60(-0.45$ to 1.66$)$ & 0.26 & $95 \%$ \\
\hline
\end{tabular}

Std. Mean Difference

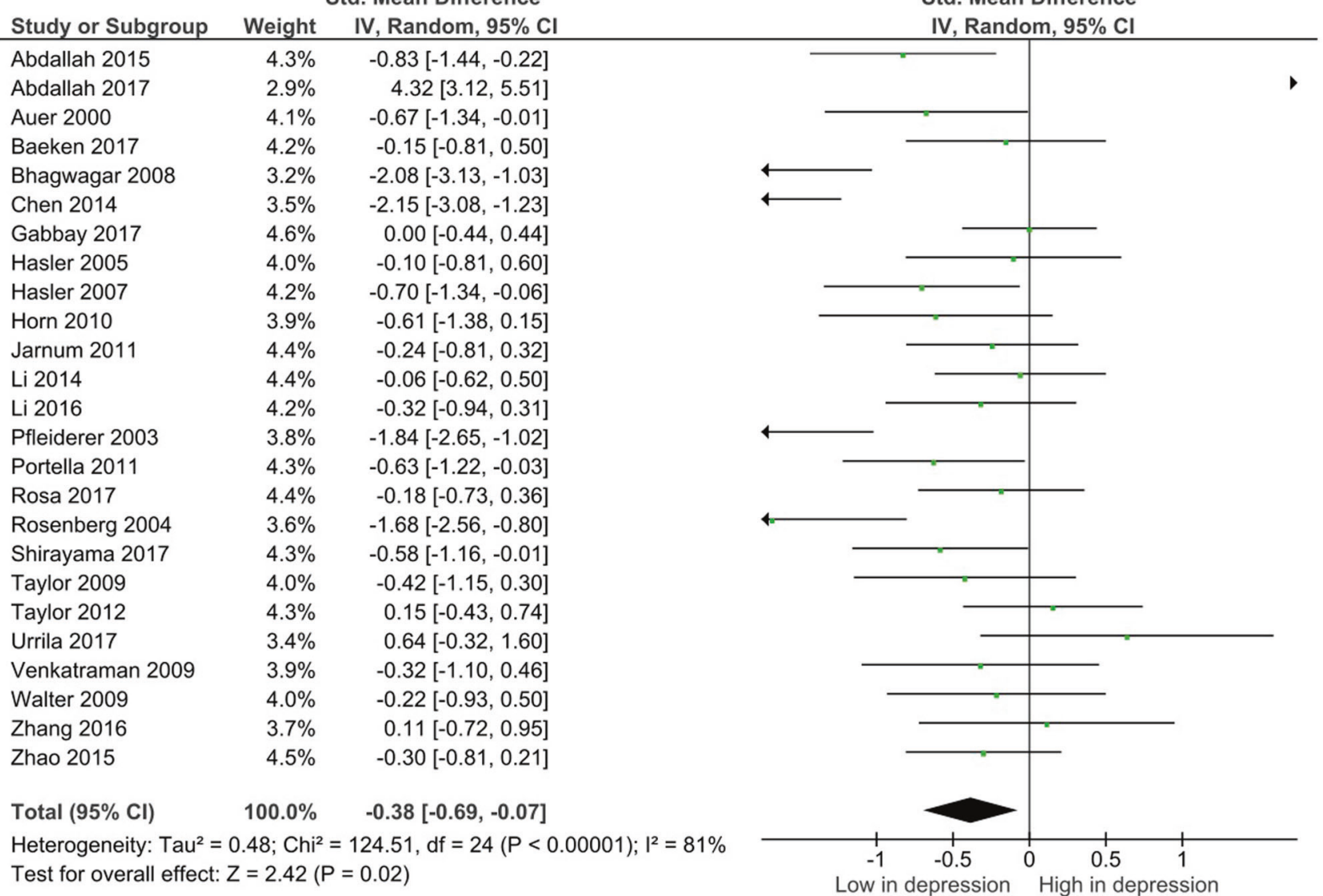

Fig. 2 Study effect sizes of Glx differences between depression and controls in the medial prefrontal cortex. Each data marker represents a study, and the size of the data marker is proportional to the total

\section{Moderator analyses}

\section{Subgroup analyses and sensitivity analysis}

Levels of Glx $\left(I^{2}=81 \%\right)$, glutamate $\left(I^{2}=85 \%\right)$, and glutamine $\left(I^{2}=95 \%\right)$ in the mPFC showed considerable heterogeneity. In addition, the leave-1-out sensitivity analysis number of individuals in that study. The summary effect size for each brain region is denoted by a diamond

showed that the results of Glx in the $\mathrm{mPFC}$ were robust. Subgroup analyses of Glx levels in the mPFC revealed that there was a significant decrease in medicated patients with depression (SMD $=-0.50 ; 95 \% \mathrm{CI},-0.80$ to -0.20 ; $\left.I^{2}=52 \% ; p=0.001\right)$, but not in unmedicated patients $\left(\mathrm{SMD}=-0.27 ; 95 \% \quad \mathrm{CI}, \quad-0.76\right.$ to $0.21 ; I^{2}=87 \%$; $p=0.27)$. The leave-1-out sensitivity analysis showed that 


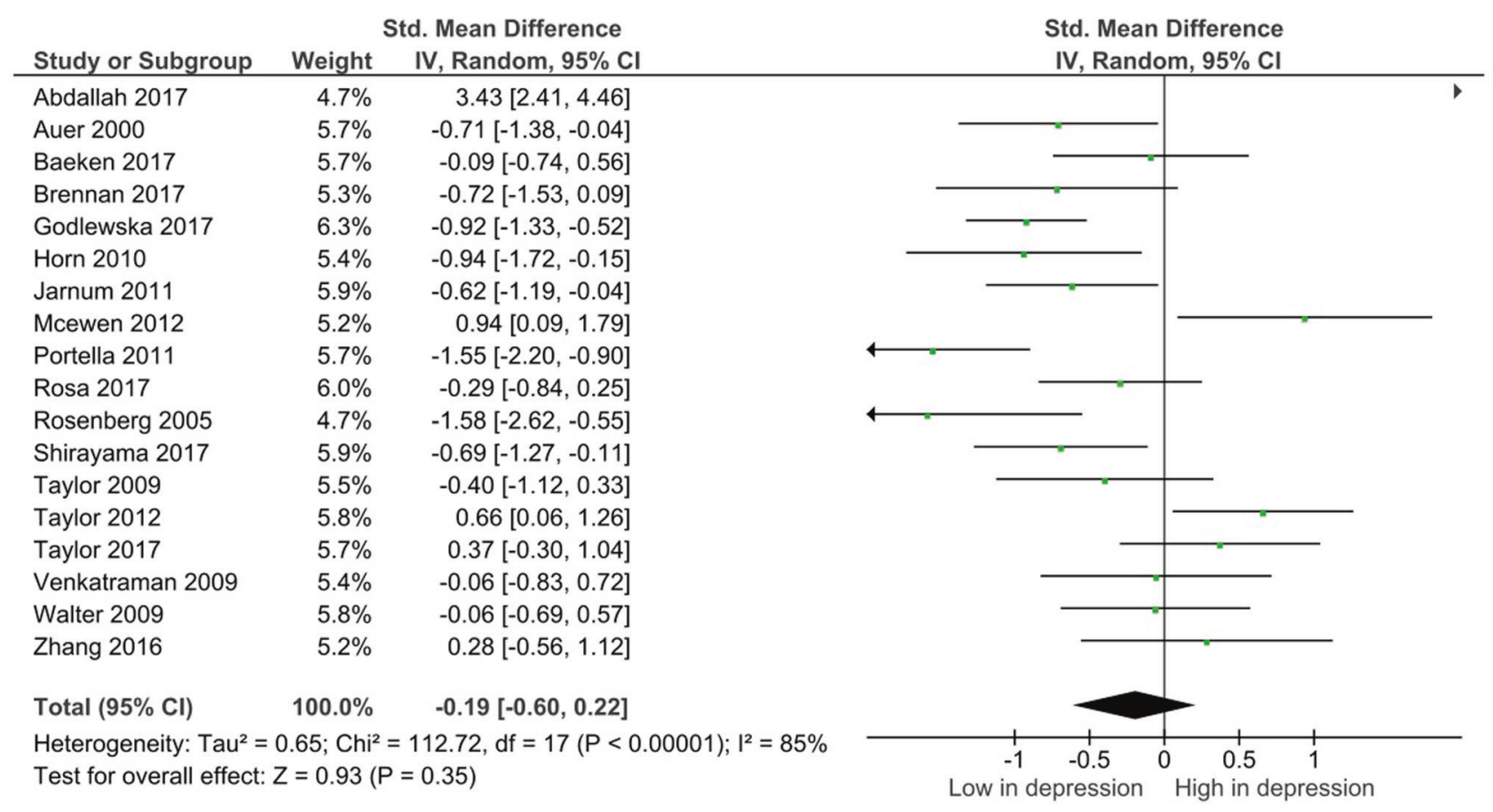

Fig. 3 Study effect sizes of glutamate differences between depression and controls in the medial prefrontal cortex. Each data marker represents a study, and the size of the data marker is proportional to the total

the SMD of one study in the unmedicated depression group was high; after removing the study, there was a significant decrease in unmedicated patients with depression [18]. We also conducted subgroup analyses on mPFC Glx levels for varying reference methods. mPFC Glx levels corrected for CSF were significantly lower in patients with depression compared with controls $(\mathrm{SMD}=-0.60 ; 95 \%$ CI, -0.93 to $-0.27 ; I^{2}=71 \% ; p<0.001$ ), whereas there was no significant difference in mPFC Glx levels between groups when Glx levels were referenced to creatine levels $(\mathrm{SMD}=-0.05 ; 95 \% \mathrm{CI},-0.63$ to $0.53 ; \mathrm{I} 2=86 \%$; $p=0.87)$.

\section{Meta-regression analyses}

There were no associations between subject age (coefficient: -0.0082 ; 95\% CI: $(-0.036,0.020) ; p=0.56)$, female ratio (coefficient: -0.26 ; 95\% CI: $(-2.0,1.5) ; p=0.77)$, or clinical severity of HAMD 17 (coefficient: $-0.0082 ; 95 \%$ CI: $(-0.070,0.042) ; p=0.62)$ and Glx levels in the mPFC.

\section{Publication bias}

The Begg-Kendall test did not indicate any publication bias for mPFC Glx or mPFC glutamate, respectively $($ tau $=-0.18, p=0.21 ;$ tau $=-0.19, p=0.27$, respectively) (Supplementary Figure 1). number of individuals in that study. The summary effect size for each brain region is denoted by a diamond

\section{Discussion}

This was the first study to compare glutamatergic neurometabolites, such as glutamate, glutamine, and Glx in broad brain regions between patients with depression and healthy controls, while also considering factors that can affect glutamatergic levels, such as medication status. We conducted a meta-analysis to compare levels of glutamatergic neurometabolites between patients with depression and controls. Our main findings are fourfold: (1) with a small effect size, Glx levels were decreased within the mPFC in patients with depression compared with controls; (2) no significant differences were found in glutamate or glutamine levels between the two groups; (3) Glx levels were lower within the mPFC in medicated patients with depression compared with controls, whereas no differences were found between unmedicated patients with depression and controls; and (4) no relationships were found between the effect sizes of mPFC Glx levels and any clinical variables in patients with depression.

\section{Main findings}

Both animal and clinical studies have proposed that glutamatergic dysfunction is implicated in the pathophysiology of depression. Animal studies have demonstrated that stress causes depressive states that are accompanied by 
glutamatergic system alterations [19]. Chronic mild stress decreases the expression of NMDA receptor subunits in the frontal cortex [20, 21]. Repeated stress also decreases the expression of $\alpha$-amino-3-hydroxy-5-methyl-4-isoxazolepropionic acid (AMPA) receptor subunits in the PFC [22]. Exposure to chronic stress decreases the number of mPFC neurons in rats [23, 24]. Thus, an extensive body of evidence has strongly indicated that the depressive state is related to abnormalities in the glutamatergic system.

Clinical studies have also suggested that the glutamatergic system plays an important role in the pathophysiology of depression. Some studies have reported that glutamate levels in plasma and cerebrospinal fluid (CSF) are higher in patients with depression than in controls [25-28], whereas others have indicated that there are no difference between the two groups [29, 30]. In addition, post-mortem studies have found higher glutamate levels in the mPFC of patients with depression than in that of controls [31]. However, in contrast, another study showed that there was a reduction of CSF glutamate levels in patients with treatment-resistant depression compared with controls [32]. Thus, the existing findings that have assessed glutamate levels in depression are inconsistent and seem to depend on the studies and sample source. These inconsistent findings may be due to the clinical and biological heterogeneity of depression. On the other hand, post-mortem studies have also noted glutamatergic dysfunction within the frontal cortex of patients with depression. There were observed reductions in the density of glutamatergic neurons in the orbitofrontal cortex of patients with depression [33]. In addition, reductions in the protein expression of NMDA receptor subunits (NR2A and NR2B) were observed in the PFC of patients with depression [20]. A PET study using ${ }^{11} \mathrm{C}$-ABP688 revealed lower mGluR5 availability in the PFC, cingulate cortex, insula, thalamus, and hippocampus in the depression group compared with the controls [4]. In another study, a lower mGluR5 availability with ${ }^{11} \mathrm{C}$ ABP688 was also detected in patients with depression compared with controls in many cortical areas [34]. These findings are corroborated by preclinical studies, suggesting that depressive symptoms may be associated with reductions in the mGluR5 protein $[35,36]$. Collectively, these findings suggest that glutamatergic mGluR dysfunction might contribute to the pathophysiology of depression. However, the physiological implications of mGluR5 disturbances in depression still remain unclear [37-39]. Again, these observations from animal and clinical studies support the hypothesis that disruption of the glutamatergic system is associated with the pathophysiology of depression, and they further suggest that modulation of the glutamatergic system may lead to a novel therapeutic approach to depression. Our main finding of Glx reduction within the $\mathrm{MPFC}$ in patients with depression warrants further studies to fully elucidate the underlying mechanism of depression and the therapeutic implications of modulating the glutamatergic system.

It has been reported that a variety of depression treatments increase Glx levels in the mPFC. For example, antidepressants and electroconvulsive therapy (ECT) increase Glx levels in the mPFC in patients with depression $[11,13$, 40]. In addition, several lines of investigation have shown that ketamine, an NMDA receptor antagonist, has antidepressant effects and increases glutamate levels in the PFC in patients with depression through NMDAreceptor inhibition and subsequent AMPAR activation [41, 42]. The NMDAreceptor inhibition and subsequent AMPAreceptor stimulation leads to inhibition of eukaryotic elongation factor 2 kinase, as well as activation of brain-derived neurotrophic factor, tropomyosin-related kinase $\mathrm{B}$, and mammalian target of rapamycin signaling, thereby increasing levels of synaptic proteins in the PFC [43-45]. Overall, these results support hypo-glutamatergic function in depression and are in line with our finding of decreased levels of Glx within the $\mathrm{mPFC}$ in patients with depression.

\section{Findings of moderator analyses}

The main finding should be confirmed in further studies because the heterogeneity of the included studies was high. Our subanalyses found that Glx levels were lower in the $\mathrm{mPFC}$ of medicated patients with depression than those of controls, whereas there were no differences between unmedicated patients with depression and controls. However, one study reported high Glx levels in unmedicated patients compared with controls [18]. After removing this study, we found that there was also a significant decrease of Glx levels in unmedicated patients with depression in comparison with controls. In addition, effects sizes were similar between unmedicated and medicated patients compared with controls. However, as described above, it has previously been reported that treatment of depression is associated with Glx elevations within the mPFC in patients with depression; this has been shown with antidepressant treatment, ECT, and ketamine administration [11, 13, 40, 42]. It is difficult to accurately assess the difference between the treatment group and the untreated group due to the high heterogeneity of the included studies in both groups. Furthermore, medication information other than antidepressants, such as benzodiazepines, mood stabilizers, and antipsychotics, was not sufficient to perform the meta-regression analyses. Thus, further research is clearly needed to elucidate the effects of various depression treatments on glutamatergic neurometabolites.

Notably, the present meta-regression did not find that glutamatergic neurometabolite concentrations in patients vary in association with symptom severity. Thus, the group difference in mPFC Glx levels between patients with depression and controls could not be explained by symptom severity. Of 
note, the symptomatology of depression, including psychotic symptoms or melancholic types, represents a clinically important factor that might also contribute to the heterogeneity. However, information concerning symptoms was insufficient in the included studies for further analyses. Thus, further studies will be required to investigate specific subtypes of depression.

Our subgroup analyses found that mPFC Glx levels corrected for CSF were lower in patients with depression compared with controls, whereas there were no group differences in $\mathrm{mPFC}$ Glx levels referenced to creatine levels. Given that creatine levels are lower in patients with depression compared with controls (Supplementary Figure 3), Glx levels referenced to creatine could be overestimated in patients with depression. Taken together, these findings again suggested that mPFC Glx levels may be decreased in patients with depression in comparison with controls.

\section{Limitations}

This meta-analysis has some limitations. First, although the meta-analysis analyzed data region by region, both voxel sizes and ROIs were different among the included studies. The quality control procedure also varied among studies. These differences might skew the results of ROI analyses. Second, the field strength of most studies was $3 \mathrm{~T}$ or lower, which precluded the accurate division of glutamate and glutamine. We could not specify which glutamatergic metabolites in which cell types are affected in depression. Glx is made up of both glutamate and glutamine but glutamate accounts for about $80 \%$ of Glx levels at $1.5 \mathrm{~T}$ or $3 \mathrm{~T}[46] .{ }^{1} \mathrm{H}$ MRS also does not provide information regarding cell types. Future studies should include studies with increased field strength to improve separation of individual signal spectra. Third, the present study did not consider confounders such as food or smoking status. The majority of studies included in the meta-analysis did not include meal or smoking information. Food intake can influence Glx levels in the brain, with Glx decreasing by as much as $17 \%$ in the PFC after fasting [47]. Smoking also interferes with the glutamate levels in the mPFC [48]. Thus, studies that report food or smoking condition should be included in future meta-analyses. Fourth, the number of included subjects in studies was relatively small. The sample sizes ranged from 9 to 63 for depression and 10-50 for healthy controls. Further studies with larger sample sizes should be conducted. Fourth, we could not analyze specific age groups even though we included studies with no exclusion in age. Indeed, a few studies investigated pediatric subjects. Thus, future studies are warranted to investigate a wide range of age groups in an effort to confirm whether the results would be consistent in both pediatric and adult patients with depression. Finally, statistical heterogeneity is high in terms of Glx in the mPFC. In the past meta-analysis, mPFC Glx levels in patients with depression were associated with symptom severity in metaregression analyses, although such a relationship was not identified in our analysis [15]. This is likely attributable to the clinical heterogeneity caused by differences in patient characteristics [49] or the influence of other methodological factors such as neurometabolite reference method (i.e., $\mathrm{Cr}$ versus water).

\section{Conclusion}

The results of this meta-analysis suggest that depression is associated with decreased levels of Glx in the mPFC. This further substantiates the development of novel treatment interventions that seek to modulate the glutamatergic system in patients with depression.

\section{Compliance with ethical standards}

Conflict of interest DMB receives research support from the Canadian Institutes of Health Research (CIHR), National Institutes of Health US (NIH), Weston Brain Institute, Brain Canada and the Temerty Family through the CAMH Foundation and the Campbell Research Institute. He received research support and in-kind equipment support for an investigator-initiated study from Brainsway Ltd. and he is the site principal investigator for three sponsor-initiated studies for Brainsway Ltd. He received in-kind equipment support from Magventure for an investigator-initiated study. He received medication supplies for an investigator-initiated trial from Indivior. He has participated in an advisory board for Janssen.

MM has received grants and/or speaker's honoraria from Asahi Kasei Pharma, Astellas Pharmaceutical, Daiichi Sankyo, Dainippon-Sumitomo Pharma, Eisai, Eli Lilly, Fuji Film RI Pharma, Janssen Pharmaceutical, Kracie, Meiji-Seika Pharma, Mochida Pharmaceutical, MSD, Novartis Pharma, Ono Yakuhin, Otsuka Pharmaceutical, Pfizer, Shionogi, Takeda Yakuhin, Tanabe Mitsubishi Pharma, and Yoshitomi Yakuhin within the past three years.

YN receives research grants from Japan Health Foundation, Meiji Yasuda Mental Health Foundation, Mitsui Life Social Welfare Foundation, Takeda Science Foundation, SENSHIN Medical Research Foundation, Health Science Center Foundation, and Daiichi Sankyo Scholarship Donation Program. He receives equipment-in-kind support for an investigator-initiated study from Magventure Inc.

ZJD has received within the last 3 years both research and equipment in-kind support for an investigator-initiated study through Brainsway Ltd. and Magventure.

EP has received funding from the Vanier Canada Graduate Scholarship, the Ontario Graduate Scholarship, and the Canada Graduate Scholarship-Master's.

AG has received support from the United States National Institute of Health, CIHR, OMHF, Consejo Nacional de Ciencia y Tecnología, the Instituto de Ciencia y Tecnología del DF, the Brain \& Behavior Research Foundation (Formerly NARSAD), the Ontario Ministry of Health and Long-Term Care, the Ontario Ministry of Research and Innovation Early Research Award, and Janssen.

NS has received fellowship grants from CIHR, research support from Japan Society for the Promotion of Science, Japan Research 
Foundation for Clinical Pharmacology, Naito Foundation, Uehara Memorial Foundation, Takeda Science Foundation, Daiichi Sankyo, and MSD, manuscript fees or speaker's honoraria from Dainippon Sumitomo Pharma and Yoshitomi Yakuhin within the past three years. Other authors have no financial or other relationship relevant to the subject of this manuscript.

TK has received consultant fees from Dainippon Sumitomo, Novartis, Otsuka and speaker's honoraria from Banyu, Eli Lilly, Dainippon Sumitomo, Janssen, Novartis, Otsuka and Pfizer. He has received grant support from the Pfizer Health Research, Takeda, Tanabe-Mitsubishi, Dainippon-Sumitomo, Otsuka and Mochida.

JHM have received operating grant funds for other studies from Janssen in the past 5 years. JHM has been a consultant to Mylan, Lundbeck, Takeda, Teva, and Trius in the past 5 years. JHM is an inventor on five patents of blood markers to predict brain inflammation or to diagnose affective disorders, and a dietary supplement to reduce depressed mood postpartum.

Open Access This article is licensed under a Creative Commons Attribution 4.0 International License, which permits use, sharing, adaptation, distribution and reproduction in any medium or format, as long as you give appropriate credit to the original author(s) and the source, provide a link to the Creative Commons license, and indicate if changes were made. The images or other third party material in this article are included in the article's Creative Commons license, unless indicated otherwise in a credit line to the material. If material is not included in the article's Creative Commons license and your intended use is not permitted by statutory regulation or exceeds the permitted use, you will need to obtain permission directly from the copyright holder. To view a copy of this license, visit http://creativecommons. org/licenses/by/4.0/

\section{References}

1. Trullas R, Skolnick P. Functional antagonists at the NMDA receptor complex exhibit antidepressant actions. Eur J Pharmacol. 1990;185:1-10.

2. Berman RM, Cappiello A, Anand A, Oren DA, Heninger GR, Charney DS, et al. Antidepressant effects of ketamine in depressed patients. Biol Psychiatry. 2000;47:351-4.

3. Lener MS, Niciu MJ, Ballard ED, Park M, Park LT, Nugent AC, et al. Glutamate and gamma-aminobutyric acid systems in the pathophysiology of major depression and antidepressant response to ketamine. Biol Psychiatry. 2017;81:886-97.

4. Deschwanden A, Karolewicz B, Feyissa AM, Treyer V, Ametamey SM, Johayem A, et al. Reduced metabotropic glutamate receptor 5 density in major depression determined by [(11)C]ABP688 PET and postmortem study. Am J Psychiatry. 2011;168:727-34.

5. Tordera RM, Totterdell S, Wojcik SM, Brose N, Elizalde N, Lasheras B, et al. Enhanced anxiety, depressive-like behaviour and impaired recognition memory in mice with reduced expression of the vesicular glutamate transporter 1 (VGLUT1). Eur J Neurosci. 2007;25:281-90.

6. Lee Y, Son H, Kim G, Kim S, Lee DH, Roh GS, et al. Glutamine deficiency in the prefrontal cortex increases depressive-like behaviours in male mice. J Psychiatry Neurosci. 2013;38:183-91.

7. Almeida RF, Thomazi AP, Godinho GF, Saute JA, Wofchuk ST, Souza DO, et al. Effects of depressive-like behavior of rats on brain glutamate uptake. Neurochem Res. 2010;35:1164-71.

8. McGirr A, Berlim MT, Bond DJ, Fleck MP, Yatham LN, Lam RW. A systematic review and meta-analysis of randomized, double-blind, placebo-controlled trials of ketamine in the rapid treatment of major depressive episodes. Psychol Med. 2015;45: 693-704.
9. Baeken C, Lefaucheur JP, Van Schuerbeek P. The impact of accelerated high frequency rTMS on brain neurochemicals in treatment-resistant depression: insights from (1)H MR spectroscopy. Clin Neurophysiol. 2017;128:1664-72.

10. Gabbay V, Bradley KA, Mao X, Ostrover R, Kang G, Shungu DC. Anterior cingulate cortex gamma-aminobutyric acid deficits in youth with depression. Transl Psychiatry. 2017;7:e1216.

11. Pfleiderer B, Michael N, Erfurth A, Ohrmann P, Hohmann U, Wolgast $M$, et al. Effective electroconvulsive therapy reverses glutamate/glutamine deficit in the left anterior cingulum of unipolar depressed patients. Psychiatry Res. 2003;122:185-92.

12. Bhagwagar Z, Wylezinska M, Jezzard P, Evans J, Boorman E, MM P, et al. Low GABA concentrations in occipital cortex and anterior cingulate cortex in medication-free, recovered depressed patients. Int J Neuropsychopharmacol. 2008;11:255-60.

13. Chen LP, Dai HY, Dai ZZ, Xu CT, Wu RH. Anterior cingulate cortex and cerebellar hemisphere neurometabolite changes in depression treatment: a $1 \mathrm{H}$ magnetic resonance spectroscopy study. Psychiatry Clin Neurosci. 2014;68:357-64.

14. Luykx JJ, Laban KG, van den Heuvel MP, Boks MP, Mandl RC, Kahn RS, et al. Region and state specific glutamate downregulation in major depressive disorder: a meta-analysis of $(1) \mathrm{H}-$ MRS findings. Neurosci Biobehav Rev. 2012;36:198-205.

15. Arnone D, Mumuni AN, Jauhar S, Condon B, Cavanagh J. Indirect evidence of selective glial involvement in glutamatebased mechanisms of mood regulation in depression: metaanalysis of absolute prefrontal neuro-metabolic concentrations. Eur Neuropsychopharmacol. 2015;25:1109-17.

16. Moher D, Liberati A, Tetzlaff J, Altman DG, Group P. Preferred reporting items for systematic reviews and meta-analyses: the PRISMA statement. BMJ. 2009;339:b2535.

17. Stang A. Critical evaluation of the Newcastle-Ottawa scale for the assessment of the quality of nonrandomized studies in metaanalyses. Eur J Epidemiol. 2010;25:603-5.

18. Abdallah CG, Hannestad J, Mason GF, Holmes SE, DellaGioia N, Sanacora G, et al. Metabotropic glutamate receptor 5 and glutamate involvement in major depressive disorder: a multimodal imaging study. Biol Psychiatry Cogn Neurosci Neuroimaging. 2017;2:449-56.

19. Pittenger C, Duman RS. Stress, depression, and neuroplasticity: a convergence of mechanisms. Neuropsychopharmacology. 2008; 33:88-109.

20. Feyissa AM, Chandran A, Stockmeier CA, Karolewicz B. Reduced levels of NR2A and NR2B subunits of NMDA receptor and PSD-95 in the prefrontal cortex in major depression. Prog Neuropsychopharmacol Biol Psychiatry. 2009;33:70-75.

21. Lou JS, Li CY, Yang XC, Fang J, Yang YX, Guo JY. Protective effect of gan mai da zao decoction in unpredictable chronic mild stress-induced behavioral and biochemical alterations. Pharm Biol. 2010;48:1328-36.

22. Yuen EY, Wei J, Liu W, Zhong P, Li X, Yan Z. Repeated stress causes cognitive impairment by suppressing glutamate receptor expression and function in prefrontal cortex. Neuron. 2012;73: 962-77.

23. Li N, Liu RJ, Dwyer JM, Banasr M, Lee B, Son H, et al. Glutamate $\mathrm{N}$-methyl-D-aspartate receptor antagonists rapidly reverse behavioral and synaptic deficits caused by chronic stress exposure. Biol Psychiatry. 2011;69:754-61.

24. Duman RS, Aghajanian GK. Synaptic dysfunction in depression: potential therapeutic targets. Science. 2012;338:68-72.

25. Altamura CA, Mauri MC, Ferrara A, Moro AR, D'Andrea G, Zamberlan F. Plasma and platelet excitatory amino acids in psychiatric disorders. Am J Psychiatry. 1993;150:1731-3.

26. Kim JS, Schmid-Burgk W, Claus D, Kornhuber HH. Increased serum glutamate in depressed patients. Arch Psychiatr Nervenkr (1970). 1982;232:299-304. 
27. Mauri MC, Ferrara A, Boscati L, Bravin S, Zamberlan F, Alecci $\mathrm{M}$, et al. Plasma and platelet amino acid concentrations in patients affected by major depression and under fluvoxamine treatment. Neuropsychobiology. 1998;37:124-9.

28. Levine J, Panchalingam K, Rapoport A, Gershon S, McClure RJ, Pettegrew JW. Increased cerebrospinal fluid glutamine levels in depressed patients. Biol Psychiatry. 2000;47:586-93.

29. Altamura C, Maes M, Dai J, Meltzer HY. Plasma concentrations of excitatory amino acids, serine, glycine, taurine and histidine in major depression. Eur Neuropsychopharmacol. 1995;5:Suppl: 71-75.

30. Maes M, Verkerk R, Vandoolaeghe E, Lin A, Scharpe S. Serum levels of excitatory amino acids, serine, glycine, histidine, threonine, taurine, alanine and arginine in treatment-resistant depression: modulation by treatment with antidepressants and prediction of clinical responsivity. Acta Psychiatr Scand. 1998;97:302-8.

31. Hashimoto K, Sawa A, Iyo M. Increased levels of glutamate in brains from patients with mood disorders. Biol Psychiatry. 2007; 62:1310-6.

32. Frye MA, Tsai GE, Huggins T, Coyle JT, Post RM. Low cerebrospinal fluid glutamate and glycine in refractory affective disorder. Biol Psychiatry. 2007;61:162-6.

33. Rajkowska G, Miguel-Hidalgo JJ, Dubey P, Stockmeier CA, Krishnan KR. Prominent reduction in pyramidal neurons density in the orbitofrontal cortex of elderly depressed patients. Biol Psychiatry. 2005;58:297-306.

34. Esterlis I, DellaGioia N, Pietrzak RH, Matuskey D, Nabulsi N, Abdallah CG, et al. Ketamine-induced reduction in mGluR5 availability is associated with an antidepressant response: an [(11) C]ABP688 and PET imaging study in depression. Mol Psychiatry. 2018;23:824-32.

35. Shin S, Kwon O, Kang JI, Kwon S, Oh S, Choi J, et al. mGluR5 in the nucleus accumbens is critical for promoting resilience to chronic stress. Nat Neurosci. 2015;18:1017-24.

36. Kovacevic T, Skelin I, Minuzzi L, Rosa-Neto P, Diksic M. Reduced metabotropic glutamate receptor 5 in the Flinders Sensitive Line of rats, an animal model of depression: an autoradiographic study. Brain Res Bull. 2012;87:406-12.

37. Belozertseva IV, Kos T, Popik P, Danysz W, Bespalov AY. Antidepressant-like effects of mGluR1 and mGluR5 antagonists in the rat forced swim and the mouse tail suspension tests. Eur Neuropsychopharmacol. 2007;17:172-9.

38. Pilc A, Klodzinska A, Branski P, Nowak G, Palucha A, Szewczyk $\mathrm{B}$, et al. Multiple MPEP administrations evoke anxiolytic- and antidepressant-like effects in rats. Neuropharmacology. 2002;43: 181-7.

39. Chaki S, Ago Y, Palucha-Paniewiera A, Matrisciano F, Pilc A. mGlu2/3 and mGlu5 receptors: potential targets for novel antidepressants. Neuropharmacology. 2013;66:40-52.

40. Njau S, Joshi SH, Espinoza R, Leaver AM, Vasavada M, Marquina A, et al. Neurochemical correlates of rapid treatment response to electroconvulsive therapy in patients with major depression. J Psychiatry Neurosci. 2017;42:6-16.

41. Zanos P, Moaddel R, Morris PJ, Georgiou P, Fischell J, Elmer GI, et al. NMDAR inhibition-independent antidepressant actions of ketamine metabolites. Nature. 2016;533:481-6.

42. Moghaddam B, Adams B, Verma A, Daly D. Activation of glutamatergic neurotransmission by ketamine: a novel step in the pathway from NMDA receptor blockade to dopaminergic and cognitive disruptions associated with the prefrontal cortex. J Neurosci. 1997;17:2921-7.

43. Li N, Lee B, Liu RJ, Banasr M, Dwyer JM, Iwata M, et al. mTORdependent synapse formation underlies the rapid antidepressant effects of NMDA antagonists. Science. 2010;329:959-64.

44. Krystal JH, Sanacora G, Duman RS. Rapid-acting glutamatergic antidepressants: the path to ketamine and beyond. Biol Psychiatry. 2013;73:1133-41.
45. Autry AE, Adachi M, Nosyreva E, Na ES, Los MF, Cheng PF, et al. NMDA receptor blockade at rest triggers rapid behavioural antidepressant responses. Nature. 2011;475:91-95.

46. Snyder J, Wilman A. Field strength dependence of PRESS timings for simultaneous detection of glutamate and glutamine from 1.5 to 7T. J Magn Reson. 2010;203:66-72.

47. Ding XQ, Maudsley AA, Schweiger U, Schmitz B, Lichtinghagen $\mathrm{R}$, Bleich $\mathrm{S}$, et al. Effects of a $72 \mathrm{~h}$ fasting on brain metabolism in healthy women studied in vivo with magnetic resonance spectroscopic imaging. J Cereb Blood Flow Metab. 2018;38:469-78.

48. Mennecke A, Gossler A, Hammen T, Dorfler A, Stadlbauer A, Rosch J, et al. Physiological effects of cigarette smoking in the limbic system revealed by 3 tesla magnetic resonance spectroscopy. J Neural Transm (Vienna). 2014;121:1211-9.

49. Ostergaard SD, Jensen SO, Bech P. The heterogeneity of the depressive syndrome: when numbers get serious. Acta Psychiatr Scand. 2011;124:495-6.

50. Abdallah CG, Jiang L, De Feyter HM, Fasula M, Krystal JH, Rothman DL, et al. Glutamate metabolism in major depressive disorder. Am J Psychiatry. 2014;171:1320-7.

51. Abdallah CG, Jackowski A, Sato JR, Mao X, Kang G, Cheema R, et al. Prefrontal cortical GABA abnormalities are associated with reduced hippocampal volume in major depressive disorder. Eur Neuropsychopharmacol. 2015;25:1082-90.

52. Auer DP, Putz B, Kraft E, Lipinski B, Schill J, Holsboer F. Reduced glutamate in the anterior cingulate cortex in depression: an in vivo proton magnetic resonance spectroscopy study. Biol Psychiatry. 2000;47:305-13.

53. Bhagwagar Z, Wylezinska M, Jezzard P, Evans J, Ashworth F, Sule A, et al. Reduction in occipital cortex gamma-aminobutyric acid concentrations in medication-free recovered unipolar depressed and bipolar subjects. Biol Psychiatry. 2007;61:806-12.

54. Binesh N, Kumar A, Hwang S, Mintz J, Thomas MA. Neurochemistry of late-life major depression: a pilot two-dimensional MR spectroscopic study. J Magn Reson Imaging. 2004;20:1039-45.

55. Block W, Traber F, von Widdern O, Metten M, Schild H, Maier $\mathrm{W}$, et al. Proton MR spectroscopy of the hippocampus at $3 \mathrm{~T}$ in patients with unipolar major depressive disorder: correlates and predictors of treatment response. Int $\mathbf{J}$ Neuropsychopharmacol. 2009;12:415-22.

56. Brennan BP, Admon R, Perriello C, LaFlamme EM, Athey AJ, Pizzagalli DA, et al. Acute change in anterior cingulate cortex GABA, but not glutamine/glutamate, mediates antidepressant response to citalopram. Psychiatry Res. 2017;269:9-16.

57. Caetano SC, Fonseca M, Olvera RL, Nicoletti M, Hatch JP, Stanley JA, et al. Proton spectroscopy study of the left dorsolateral prefrontal cortex in pediatric depressed patients. Neurosci Lett. 2005;384:321-6.

58. de Diego-Adelino J, Portella MJ, Gomez-Anson B, LopezMoruelo O, Serra-Blasco M, Vives Y, et al. Hippocampal abnormalities of glutamate/glutamine, $\mathrm{N}$-acetylaspartate and choline in patients with depression are related to past illness burden. $\mathbf{J}$ Psychiatry Neurosci. 2013;38:107-16.

59. Godlewska BR, Near J, Cowen PJ. Neurochemistry of major depression: a study using magnetic resonance spectroscopy. Psychopharmacol (Berl). 2015;232:501-7.

60. Godlewska BR, Masaki C, Sharpley AL, Cowen PJ, Emir UE. Brain glutamate in medication-free depressed patients: a proton MRS study at 7 Tesla. Psychol Med 2018;48:1731-7.

61. Grimm S, Luborzewski A, Schubert F, Merkl A, Kronenberg G, Colla M, et al. Region-specific glutamate changes in patients with unipolar depression. J Psychiatr Res. 2012;46:1059-65.

62. Hasler G, Neumeister A, van der Veen JW, Tumonis T, Bain EE, Shen J, et al. Normal prefrontal gamma-aminobutyric acid levels in remitted depressed subjects determined by proton magnetic resonance spectroscopy. Biol Psychiatry. 2005;58:969-73. 
63. Hasler G, van der Veen JW, Tumonis T, Meyers N, Shen J, Drevets WC. Reduced prefrontal glutamate/glutamine and gamma-aminobutyric acid levels in major depression determined using proton magnetic resonance spectroscopy. Arch Gen Psychiatry. 2007;64:193-200.

64. Hermens DF, Chitty KM, Lee RS, Tickell A, Haber PS, Naismith SL, et al. Hippocampal glutamate is increased and associated with risky drinking in young adults with major depression. J Affect Disord. 2015;186:95-98.

65. Horn DI, Yu C, Steiner J, Buchmann J, Kaufmann J, Osoba A, et al. Glutamatergic and resting-state functional connectivity correlates of severity in major depression - the role of pregenual anterior cingulate cortex and anterior insula. Front Syst Neurosci. 2010;4:pii: 33.

66. Jarnum H, Eskildsen SF, Steffensen EG, Lundbye-Christensen S, Simonsen CW, Thomsen IS, et al. Longitudinal MRI study of cortical thickness, perfusion, and metabolite levels in major depressive disorder. Acta Psychiatr Scand. 2011;124:435-46.

67. Jayaweera HK, Lagopoulos J, Duffy SL, Lewis SJ, Hermens DF, Norrie L, et al. Spectroscopic markers of memory impairment, symptom severity and age of onset in older people with lifetime depression: discrete roles of $\mathrm{N}$-acetyl aspartate and glutamate. $\mathrm{J}$ Affect Disord. 2015;183:31-38.

68. Jollant F, Near J, Turecki G, Richard-Devantoy S. Spectroscopy markers of suicidal risk and mental pain in depressed patients. Prog Neuropsychopharmacol Biol Psychiatry. 2017;73:64-71.

69. Li H, Xu H, Zhang Y, Guan J, Zhang J, Xu C, et al. Differential neurometabolite alterations in brains of medication-free individuals with bipolar disorder and those with unipolar depression: a two-dimensional proton magnetic resonance spectroscopy study. Bipolar Disord. 2016;18:583-90.

70. Li M, Metzger CD, Li W, Safron A, van Tol MJ, Lord A, et al. Dissociation of glutamate and cortical thickness is restricted to regions subserving trait but not state markers in major depressive disorder. J Affect Disord. 2014;169:91-100.

71. McEwen AM, Burgess DT, Hanstock CC, Seres P, Khalili P, Newman SC, et al. Increased glutamate levels in the medial prefrontal cortex in patients with postpartum depression. Neuropsychopharmacology. 2012;37:2428-35.

72. Menke A, Samann P, Kloiber S, Czamara D, Lucae S, Hennings J, et al. Polymorphisms within the metabotropic glutamate receptor 1 gene are associated with depression phenotypes. Psychoneuroendocrinology. 2012;37:565-75.

73. Michael N, Erfurth A, Ohrmann P, Arolt V, Heindel W, Pfleiderer B. Metabolic changes within the left dorsolateral prefrontal cortex occurring with electroconvulsive therapy in patients with treatment resistant unipolar depression. Psychol Med. 2003;33:1277-84.

74. Milne A, MacQueen GM, Yucel K, Soreni N, Hall GB. Hippocampal metabolic abnormalities at first onset and with recurrent episodes of a major depressive disorder: a proton magnetic resonance spectroscopy study. Neuroimage. 2009;47:36-41.

75. Mu J, Xie P, Yang ZS, Yang DL, Lv FJ, Luo TY, et al. 1H magnetic resonance spectroscopy study of thalamus in treatment resistant depressive patients. Neurosci Lett. 2007;425:49-52.

76. Nery FG, Stanley JA, Chen HH, Hatch JP, Nicoletti MA, Monkul ES, et al. Normal metabolite levels in the left dorsolateral prefrontal cortex of unmedicated major depressive disorder patients: a single voxel (1)H spectroscopy study. Psychiatry Res. 2009;174: 177-83.

77. Poletti S, Locatelli C, Falini A, Colombo C, Benedetti F. Adverse childhood experiences associate to reduced glutamate levels in the hippocampus of patients affected by mood disorders. Prog Neuropsychopharmacol Biol Psychiatry. 2016;71:117-22.
78. Portella MJ, de Diego-Adelino J, Gomez-Anson B, MorganFerrando R, Vives Y, Puigdemont D, et al. Ventromedial prefrontal spectroscopic abnormalities over the course of depression: a comparison among first episode, remitted recurrent and chronic patients. J Psychiatr Res. 2011;45:427-34.

79. Rosa CE, Soares JC, Figueiredo FP, Cavalli RC, Barbieri MA, Schaufelberger MS, et al. Glutamatergic and neural dysfunction in postpartum depression using magnetic resonance spectroscopy. Psychiatry Res. 2017;265:18-25.

80. Rosenberg DR, Mirza Y, Russell A, Tang J, Smith JM, Banerjee $\mathrm{SP}$, et al. Reduced anterior cingulate glutamatergic concentrations in childhood OCD and major depression versus healthy controls. J Am Acad Child Adolesc Psychiatry. 2004;43:1146-53.

81. Rosenberg DR, Macmaster FP, Mirza Y, Smith JM, Easter PC, Banerjee SP, et al. Reduced anterior cingulate glutamate in pediatric major depression: a magnetic resonance spectroscopy study. Biol Psychiatry. 2005;58:700-4.

82. Sanacora G, Gueorguieva R, Epperson CN, Wu YT, Appel M, Rothman DL, et al. Subtype-specific alterations of gammaaminobutyric acid and glutamate in patients with major depression. Arch Gen Psychiatry. 2004;61:705-13.

83. Shirayama Y, Takahashi M, Osone F, Hara A, Okubo T. Myoinositol, glutamate, and glutamine in the prefrontal cortex, hippocampus, and amygdala in major depression. Biol Psychiatry Cogn Neurosci Neuroimaging. 2017;2:196-204.

84. Taylor MJ, Selvaraj S, Norbury R, Jezzard P, Cowen PJ. Normal glutamate but elevated myo-inositol in anterior cingulate cortex in recovered depressed patients. J Affect Disord. 2009;119: 186-9.

85. Taylor MJ, Godlewska BR, Norbury R, Selvaraj S, Near J, Cowen PJ. Early increase in marker of neuronal integrity with antidepressant treatment of major depression: $1 \mathrm{H}$-magnetic resonance spectroscopy of N-acetyl-aspartate. Int J Neuropsychopharmacol. 2012;15:1541-6.

86. Taylor R, Osuch EA, Schaefer B, Rajakumar N, Neufeld RW, Theberge J, et al. Neurometabolic abnormalities in schizophrenia and depression observed with magnetic resonance spectroscopy at 7 T. BJPsych Open. 2017;3:6-11.

87. Urrila AS, Hakkarainen A, Castaneda A, Paunio T, Marttunen M, Lundbom N. Frontal cortex myo-inositol is associated with sleep and depression in adolescents: a proton magnetic resonance spectroscopy study. Neuropsychobiology. 2017;75:21-31.

88. Venkatraman TN, Krishnan RR, Steffens DC, Song AW, Taylor WD. Biochemical abnormalities of the medial temporal lobe and medial prefrontal cortex in late-life depression. Psychiatry Res. 2009; 172:49-54.

89. Walter M, Henning A, Grimm S, Schulte RF, Beck J, Dydak U, et al. The relationship between aberrant neuronal activation in the pregenual anterior cingulate, altered glutamatergic metabolism, and anhedonia in major depression. Arch Gen Psychiatry. 2009; 66:478-86

90. Yang XR, Langevin LM, Jaworska N, Kirton A, Lebel RM, Harris $\mathrm{AD}$, et al. Proton spectroscopy study of the dorsolateral prefrontal cortex in youth with familial depression. Psychiatry Clin Neurosci. 2016;70:269-77.

91. Zhang X, Tang Y, Maletic-Savatic M, Sheng J, Zhang X, Zhu Y, et al. Altered neuronal spontaneous activity correlates with glutamate concentration in medial prefrontal cortex of major depressed females: an fMRI-MRS study. J Affect Disord. 2016; 201:153-61.

92. Zhao LL, Xiao H, Gao XL, Wang RJ, Liu RC, Zhang P. H-1MRS analysis for prefrontal cortex, hippocampus and thalamus of adolescent patients with depression. J Med Imag Health In. 2015;5:1229-32. 